Revue

Revue de l'histoire des religions

del'histoire des religions

L'Ordre chrétien médiéval entre le droit et la foi

\title{
Introduction. De la raison à la foi : l'entrée du droit en religion
}

Laurent Mayali

(2) OpenEdition

Journals

Édition électronique

URL : http://journals.openedition.org/rhr/7818

DOI : $10.4000 /$ rhr.7818

ISSN : 2105-2573

Éditeur

Armand Colin

Édition imprimée

Date de publication : 1 décembre 2011

Pagination : 475-482

ISBN : 978-2200-92722-6

ISSN : 0035-1423

Référence électronique

Laurent Mayali, «Introduction. De la raison à la foi : I'entrée du droit en religion », Revue de I'histoire des religions [En ligne], 4 | 2011, mis en ligne le 20 février 2012, consulté le 22 septembre 2020. URL http://journals.openedition.org/rhr/7818 ; DOl : https://doi.org/10.4000/rhr.7818 


\section{Introduction De la raison à la foi : l'entrée du droit en religion}

Affirmer, comme le fait Gratien dès les premières lignes du Décret, que «Le genre humain est régi à la fois par le droit naturel et par les mœurs», traduit le souci de penser dans le champ juridique le dualisme du spirituel et du temporel qui gouverne la société médiévale. «On peut comprendre clairement», ajoute-t-il, «comment la loi divine et la loi humaine diffèrent car tout ce qui est moral est compris dans la loi divine ou loi naturelle alors que la loi humaine représente les mœurs mises par écrit et transmises comme droit» ${ }^{1}$. On sait combien, chez Gratien, l'intelligence théologique des normes prime sur le raisonnement purement juridique, mais cette sensibilité, en accord avec la culture religieuse de son temps, n'exclut pas une vision du droit de l'Église comme système de gouvernement de la société chrétienne. Pour ce faire, le droit s'intègre dans un espace normatif où, comme l'observe Pierre Legendre, l'économie du salut associe le désir du sujet au devenir de l'institution ${ }^{2}$. Tout, dans le choix des sources, l'agencement des textes et leur interprétation, participe d'une telle conception. Euvre originale par ses objectifs, l'harmonisation des canons dissonants transpose ainsi dans le droit l'unité de la foi en plaçant, au sommet d'une hiérarchie normative, le droit naturel, d'origine divine ${ }^{3}$, qui

1. Gratien, Décret, dictum, post Distinctio 1. c. 1.

2. Pierre Legendre, L'amour du censeur. Essai sur l'ordre dogmatique, Paris, 1974, p. 143-164, et du même auteur, Nomenclator. Sur la question dogmatique en Occident, II, Paris, 2006 p. 163-183 et 207-229.

3. Jean le Teutonique et Barthélémy de Brescia, Glose ordinaire au Décret, 
«prime sur tous les autres droits en raison de sa dignité et de son ancienneté $»^{4}$. Ce droit est «le droit de la Loi, celui des prophètes, des évangiles et des apôtres, c'est-à-dire ce qui est compris dans l'Ancien et le Nouveau Testaments » ${ }^{5}$. L'importance du droit humain dont les règles, écrites ou orales, forment l'essentiel des mœurs «dans leur acception la plus large», n'est pas pour autant négligée dans la mesure où il représente aussi l'ensemble des activités humaines ${ }^{6}$. C'est pourquoi l'interprétation de textes contradictoires est distinctement facilitée par une argumentation juridique qui, dès la seconde version du Décret, accompagne l'exégèse des textes sacrés et des auctoritates religieuses. Cette influence bénéficie des apports du droit romain, dont de nombreux principes viennent étayer la transformation des normes religieuses en règles de droit. Elle se concrétise dans la conception puis l'élaboration d'un ordre juridique agencé sur les rapports entre règles, d'origine et d'autorité différentes.

La juridicisation du religieux affecte en retour la représentation médiévale des règles juridiques. Elle confère au droit une légitimité renouvelée, dans le cadre d'un modèle normatif chrétien conforme au plan divin, et lui reconnaît l'autorité d'un savoir universel qui le distingue de la connaissance ordinaire des usages et pratiques coutumières suivis par les membres des divers groupes sociaux et communautés politiques ${ }^{7}$. Cette conclusion s'impose notamment

Dist. 1 c. 1, naturel: «c'est-à-dire divin»; Par les mours: «c'est-à-dire le droit coutumier ou le droit humain qu'il soit écrit ou non écrit, Causa 25 quaestio 2.»

4. Gratien, Décret, dictum ante Dist. 5 c. 1.

5. Huguccio Pisanus, Summa Decretorum, vol. I: Distinctiones I-XX, éd. Oldrich Prerovsky, Cité du Vatican, 2006, p. 13. Dist. 1 c. 1, naturel: «c'est-à-dire divin.»

6. Huguccio, op. cit.: Dist. 1 c. 1, Les mœeurs: «c'est-à-dire le droit humain, qu'il soit écrit ou non écrit. Par ce terme on comprend tout ce qui est le droit humain, c'est-à-dire fait par l'homme selon le droit divin, qu'il soit mis par écrit ou non. C'est ce que l'on comprend comme les mours au sens large.» Voir aussi, Jean le Teutonique, Glose ordinaire, Dist. 1 c. 4, Des mœenrs. «Tu peux dire que les termes "mœurs", "mœurs" (au pluriel) et "coutume" sont utilisés de manière différentes; "mœurs" est utilisé pour désigner le droit non écrit et "coutume" est généralement utilisé pour désigner le droit qu'il soit écrit ou non écrit comme dans le prochain paragraphe; et "mœurs" (pluriel) désigne fréquemment les actions humaines.».

7. Baldus de Ubaldis, Ad tres priores libros decretalium commentaria, Lyon, 1585, réimp. Aalen, 1970, fol. $3 \mathrm{vb}$ : «le caractère sacré du droit canonique est rehaussé par la grandeur du droit civil, de même que la majesté du droit civil est 
avec la redéfinition du droit canonique comme un élément du droit $\operatorname{divin}^{8}$, mais elle trouve aussi place, au versant séculier, dans l'argumentation des civilistes qui n'hésitent pas à affirmer que «tout est dans le droit», pour conclure que le jurisperitus peut donc à juste titre prétendre au savoir du théologien. Cette assertion péremptoire actualise la notion romaine de la jurisprudentia qu'Ulpien avait définie comme la connaissance des causes divines et humaines ${ }^{9}$, tout en intégrant dans le champ du droit la représentation du profane et du sacré comme mesure ultime de l'autorité des lois civiles. À vrai dire, ces arguments, pour le moins singuliers dans le contexte intellectuel du XIII ${ }^{\text {e }}$ siècle, adressent plusieurs enjeux sans doute attisés par les querelles doctrinales et les conflits politiques qui marquent le développement des premiers studia (notamment l'interdit pontifical d'enseigner le droit romain à Paris: Honorius III, Bulle Super specula[m], 1219), mais ils illustrent également une polémique qui reproduit dans ses grandes lignes l'argumentation fondée sur la dualité normative chère aux canonistes. Pour ces clercs-juristes, le droit assume une indéniable vocation spirituelle dans la mesure où il participe de l'économie du salut. «Et notre droit», conclut le cardinal d'Ostie dans ses leçons aux Décrétales, «peut vraiment prétendre être la science des sciences, car on peut par elle traiter aussi bien des questions spirituelles que temporelles ${ }^{10}$.

Dans tous les cas, l'entrée du droit en religion bouleverse la perception du rôle respectif de ces deux systèmes dans la société civile, tout en renouvelant les termes de leurs rapports mutuels dans une perspective légaliste. Certes, le phénomène n'était pas nouveau. À partir du $\mathrm{IV}^{\mathrm{e}}$ siècle, les valeurs chrétiennes avaient partiellement investi le droit romain, tout en altérant la référence antique à la sacralité des constitutions impériales et la divinité de la «loi vivante (lex animata)». Mais l'essentiel des règles et opinions rassemblées deux siècles plus tard dans les compilations de Justinien constituait encore un corpus juridique distinct de la

renforcée par l'autorité des canons de telle sorte que l'un jette des éclairs grâce à l'autre et n'en est que plus redouté.»

8. Huguccio, Summa, op. cit.: «On dit aussi que le droit divin est le droit canonique ou ecclésiastique.»

9. Digeste 1.10, Ulpien (fin $\mathrm{II}^{\mathrm{e}}$-début $\mathrm{III}^{\mathrm{e}}$ siècle A.D.).

10. Hostiensis, Summa Aurea, Lyon, 1556, fol. 3rb. 
culture religieuse byzantine. Par ailleurs, d'aucuns ont souhaité voir, de manière sans doute exagérée, la naissance de l'État moderne dans les réformes qui, tout au long $\mathrm{du} \mathrm{x}^{\mathrm{e}}$ siècle en Occident, dictent l'évolution des institutions ecclésiastiques et du pouvoir pontifical notamment illustré par les initiatives du pape Grégoire VII. Il faut observer, une fois encore, que les sphères religieuses et juridiques conservent une forte autonomie dans l'élan réformateur où l'accent mis sur la discipline ecclésiastique porte plus sur l'affirmation des valeurs pastorales que sur la mise en place d'un ordre institutionnel.

$\mathrm{Au} \mathrm{XII}{ }^{\mathrm{e}}$ siècle, cependant, l'interaction du droit et de la religion produit des conséquences autrement décisives quand elle modifie les modalités d'expression de la croyance dans son double rapport à l'être et au devoir-être et jette ainsi les fondations d'un nouvel ordre chrétien. Elle se solde notamment par l'ajout de la notion de sujet de droit à celle plus ancienne du fidèle, réunis dans une seule croyance sanctionnée par la loi tant en droit romain ${ }^{11}$ qu'en droit canonique ${ }^{12}$. L'unité de la foi trouve alors confirmation dans l'expression juridique de son caractère nécessaire et obligatoire. "Cette loi nous dit quelle foi nous devons observer, nous récompense quand nous l'observons, et nous punit quand nous ne l'observons pas», soulignent les commentaires sur l'Édit de Thessalonique qui ouvre la collection des constitutions impériales du Code de Justinien ${ }^{13}$. Il ne fait aucun doute pour le juriste médiéval que l'observance de la foi participe ainsi du respect de la loi et des institutions qui la représentent. La croyance religieuse se voit ainsi interprétée dans la formation d'un lien juridique qui unit l'individu à l'institution.

Concrètement, l'exégèse des Constitutions romaines et des Décrétales, permet d'identifier et de confirmer la corrélation des sphères privée et publique qui délimitent les champs respectifs

11. Édit de Thessalonique, Cunctos Populos, Cod. 1.1.1: «Nous ordonnons que tous les peuples soumis à notre puissance adoptent la religion que le saint apôtre Pierre a apportée aux Romains... Nous ordonnons que ceux qui suivront cette loi soient appelés chrétiens catholiques, les autres ne sont que des fous et des insensés. »

12. Liber X 1.1.1, canon Firmiter credimus (concile de Latran, 1215).

13. Jacopo de Butrio, Lectura super Codice, Paris, 1516, réimp. Bologne, 1973, fol. 4va et aussi Cino de Pistoia, In codicem commentaria, Francfort sur le Main, 1578, réimp. Turin, 1964, fol. 1A-2A. 
de la pastorale et du gouvernement. Mais l'articulation de ces deux sphères sur le mode de la croyance ne répond pas simplement au besoin politique d'affermir le pouvoir des structures institutionnelles, laïques ou ecclésiastiques. Elle est aussi chevillée dans l'idée de la complémentarité de la foi et de la raison. Alors prend forme l'idée d'une rationalité juridique qui mesure la légalité des actes et des décisions humaines à l'aune de cette double référence ${ }^{14}$. Sans pour autant être posée comme principe absolu, la raison juridique uniformise les divers espaces normatifs qui affectent les comportements identitaires individuels et collectifs. C'est pourquoi le débat sur l'essence du droit et son origine naturelle s'oriente vers la question de l'autorité des lois. Il emporte la sécularisation de la raison naturelle transposée hors de la sphère originale du droit naturel/divin ${ }^{15}$ dans celle des lois et des coutumes humaines.

Des nombreuses définitions de la raison et de la foi qui nourrissent les controverses tout au long du Moyen Âge, les juristes ne retiennent qu'une version réductrice destinée à objectiver leur valeur normative dans un système hiérarchique de règles. Cette approximation conceptuelle conduit singulièrement à les envisager sous l'angle de leur causalité. De faculté cognitive, la raison, note Ennio Cortese, assume progressivement une fonction créatrice d'effet ${ }^{16}$. Qu'elle soit définie comme ratio scripta ou qu'elle soit plus simplement évaluée en fonction des critères du raisonnable, la loi ne peut être dissociée de la raison ${ }^{17}$. Elle s'en imprègne et en devient la gardienne. Quant à la foi, elle prend valeur normative

14. Glose ordinaire, Décret, Dist. 1 c. 5 , Conforme à la religion: «Beaucoup de choses contraires à la raison sont néanmoins conformes à la religion comme le fait que la Vierge ait enfanté.»

15. Glose ordinaire, Décret, Dist. 1 c. 7, Qu'est ce que le droit naturel? union: «Si tu comprends ce texte comme traitant de l'union de leurs corps, il s'agit donc $\mathrm{du}$ droit naturel qui procède du désir physique. Si tu l'interprètes comme traitant de l'union de leurs âmes, il s'agit du droit naturel qui procède de la raison. Voir X. 1.21.5.»

16. Ennio Cortese, La Norma giuridica. Spunti teorici nel diritto comune classico, 2 vol., Milan, 1962, I, p. 42 et sq.; Peter Leisching, «Ratio in der Kanonistik », Zeitschrift der Savigny Stiftung für Rechtsgeschichte, kan. Abt 103, 1986, p. 329-337.

17. Glose ordinaire, Décret, Dist. 1 c. 5 , raison: «Donc on peut dire qu'en l'absence d'une loi ou d'un canon on peut agir selon la raison D. 19 c. 1 et D. 8 c. 7.» 
comme équivalence des bonnes mœurs ${ }^{18}$, de même que les «lois les plus sacrées » de l'ancienne nomenclature romaine désignent les lois les plus rationnelles.

Dans le même temps, l'omniprésence de la référence chrétienne dans le champ juridique produit une triple conséquence. D'une part, elle conduit à repenser le concept romain de droit public dans son rapport à la religion ${ }^{19}$. Placer «les choses sacrées et le statut des prêtres» sous son autorité comme l'enseignaient les jurisconsultes romains, signifie, pour le juriste médiéval, que «la rémission des péchés qui définit la mission de l'Église et celle des clercs relève aussi du droit public $»^{20}$. Pour les canonistes, en revanche, cette définition apporte la confirmation que toute offense contre les clercs et contre l'Église ne peut être qu'un crime public $^{21}$. À l'inscription de la pastorale dans le droit fait pendant la criminalisation des transgressions contre la religion confirmant ainsi son statut juridique. D'autre part, le développement de la procédure judiciaire romano-canonique, qui accompagne la mise en place des tribunaux ecclésiastiques aux compétences étendues hors du champ strictement religieux, fixe le lien entre justice divine («Nihil aliud est justicia quam Deus») et droit processuel dans l'autorité accordée aux jugements («Res judicata pro veritate accipitur»). Le nouvel ordre du procès reproduit les étapes du jugement divin qui, dans le récit de la Genèse, sanctionnait la faute originelle. La comparaison s'impose d'elle-même. Elle date l'origine de la procédure judiciaire de l'apparition des premières «créatures rationnelles», distinguant ainsi l'homme de l'animal non seulement dans la recherche méthodique du juste, mais aussi dans son commerce avec Dieu, source de toute justice. Au terme

18. Gratien, Décret, Dist. 11 c. 6, dictum ante: «La coutume qui n'empiète pas sur la foi est louable. Nous louons la coutume quand elle est connue pour ne pas empiéter sur la foi catholique (Décrétale de Pius I)», Glose ordinaire, ibid., foi catholique: «c'est-à-dire contre les bonnes mœurs. Pour montrer que cela est interprété dans ce sens voir De consecratione D. 4 c. 72 où l'on donne la même interprétation»; voir aussi Dist. 12 c. 11, dictum ante; Huguccio, Summa, op. cit., D. 11 c. 6 (p. 185) contre la foi: «contre les bonnes et honnêtes mœurs, voir infra Dist. 12, omnia contra», et Dist. $12 \mathrm{c} .4$, elle ne s'opposent pas à la foi (p. 199).

19. Dig. 1.1.2: «Le droit public comprend les choses sacrées, le statut des prêtres et des magistrats.»

20. Accurse, Glose ordinaire, Dig. 1.1.2, in sacris.

21. Glose ordinaire, Décret, Dist. 1. c. 8, ius publicum. 
de ce remodelage, la structure ordonnatrice des diverses phases du procès fixe un modèle de justice qui emprunte son idéal à l'exemple divin tout en mettant l'accent sur la rectitude procédurale et le respect des règles. Juger justement (juste) c'est aussi juger correctement (recte) selon le droit.

Enfin, l'interprétation de cette religion du droit (religio iuris) se solde par l'inscription de la vérité dans le corps des lois et des coutumes. «Le Seigneur a dit "Je suis la vérité". Il n'a pas dit "Je suis la coutume" $\gg^{22}$. L'antithèse coutume/vérité énoncée par Augustin puis reprise par Gratien trouve son plein emploi dans la conversion des usages et pratiques quotidiennes en droit coutumier. Elle contribue à définir la coutume comme règle de droit soumise à l'autorité de la raison et de la foi. La prégnance de la vérité comme source d'autorité dans l'Église, observe Brian Tierney ${ }^{23}$, est un phénomène largement attesté, mais son extension aux pratiques coutumières ordonne le champ juridique selon un double principe d'inclusion et de subsidiarité. Il s'agit ici moins d'exclure les coutumes que de normaliser les multiples usages et actions humaines, fixées dans la mémoire collective. Pour ce faire, raison et vérité sont associées dans le concept juridique d'une «vérité rationnelle» qui, ajoutée à la «loi rationnelle», fixe les critères objectifs de la légalitée ${ }^{4}$. Ce renvoi éclaire et complète toute discussion ultérieure de la force obligatoire de la coutume face aux lois et aux canons ${ }^{25}$. Il assure, en outre, la prégnance de la croyance, comme acte de foi et comme acte de parole, publiquement sanctionnée, dans la construction de l'ordre social. Cette approche permet d'assurer la cohérence d'un système de droit à vocation universelle, qui harmonise les exigences des sphères spirituelle et temporelle, tout en reconnaissant la

22. Gratien, Décret, Dist. 8 c. 6 : «Donc la vérité est manifeste et il faut que la coutume s'efface devant la vérité, parce que la raison et la vérité prévalent toujours sur la coutume.»

23. Brian Tierney, " "Only the Truth has authority": The problem of "reception" in the Decretists and in Johannes de Turrecremata», Law, Church and Society. Essays in honor of Stephan Kuttner, éd. Kenneth Pennington et Robert Somerville, Philadelphia, 1977, p. 69-96.

24. Summa Parisiensis, Dist. 8 c. 4 , raison et vérité: «c'est-à-dire par la vérité établie par la raison c'est-à-dire par la vérité rationnelle», éditée par Terence McLaughlin, The Summa Parisiensis on the Decretum Gratiani, Toronto, 1952, p. 8; voir aussi Huguccio, Summa, op. cit., p. 138.

25. Summa Parisiensis, op. cit., Dist. 1 c. 5, raison: «c'est-à-dire la coutume rationnelle qui devient la loi et qui a force de loi.» 
diversité des législations séculières et de pratiques coutumières hétérogènes ${ }^{26}$. À ce stade, le pluralisme juridique s'efface au profit d'un syncrétisme normatif qui promeut la stabilité des lois dans une société où la personne chrétienne prend place entre la bestialité de l'animal et la folie de l'incroyant.

lmayali@law.berkeley.edu

26. Gratien, Décret, Dist. 10 c. 1, Glose ordinaire, lois humaines: «Mais comme Justinien était vraiment un empereur catholique, il est surprenant qu'il ait légiféré contre les lois du Seigneur, car le Seigneur dit qu'une femme ne peut être séparée de son mari uniquement pour cause de fornication (Mat. 5. 32) C. 33 q. 2. c. 18. Mais tu peux dire, et certains l'affirment, qu'il l'avait permis seulement par ignorance et il mérite donc quelque indulgence. Dist. 8 c. 8 . Ou alors il s'est trompé comme Jérôme à la Dist. 26 c. 1 . Ou il l'a permis de manière à éviter un plus grand mal, de la même manière que l'usure peut être permise pour éviter le vol, de même que le divorce peut être permis pour éviter le meurtre de l'épouse, C. 33 q. 2. c. 9 .» 